\title{
RC Beam Pure Bending Plastic Hinge Parameter Calculation Considering Size Effect and Experimental Research
}

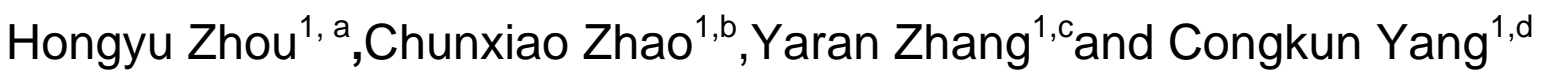 \\ ${ }^{1}$ Beijing University of Technology, Beijing 100124, P.R. China \\ azhouhy@bjut.edu.cn, b940292452@qq.com, 'cmemory15603@qq.com, ${ }^{\text {d } 15127505961 @ 163 . c o m ~}$
}

Keywords:RC beam;pure bending; plastic hinge; parameter calculation;size effect

Abstract:In order to study the influence of different loadsand cross section size on RC beam plastic hinge, a total of 13 reinforced concrete beamswith 5 section sizeswere tested under monotonic loading, and the calculation of pure bending plastic hinge parameters considering size effect isstudied based on experimental data.The results show that the plastic rotation capacity of plastic hinge zone exists significant size effect, and a way to calculate inelastic curvature and inelastic equivalent corner is put forward, measured data and calculated results are in good agreement.

\section{Introduction}

Reinforced concrete structure generates redistribution of internal forces after plastic hinges occur, Seismic structure also allows the plastic hinges appear in specific parts to dissipate seismic energy. Thus, the plastic hinge theory is the basic theory of nonlinear structure analysis.Previous studies on plastic hinge properties are mainly based on the small size results ${ }^{[1-7]}$ and the existing plastic hinge theory mainly evaluatesthe plastic deformationcapacity on steel yield phase. According to the measured results of this paper, between the large-size components ultimate bearing capacity $M_{u s, t}$ and the maximum carrying capacity $M_{\max , t}$, there is a certain strength and ductility for growth, it is more appropriate to evaluate plastic hinge propertiesin both states. The subscript here means: usultimate limit state; max- peak load state afer the specimen beyond the ultimate bearing capacity ;t-test.

\section{Test Survey and Phenomenon}

Combined with similarity relations, five groups of simply supported beam specimen of different sizes were applied grading monotonic loading until the specimen damaged ${ }^{[8-10]}$, specimen numbers were from JD1 to JD5, and pure bending zones were the main section of test observations.Extensometersweredensely placedin pure bending section of simply supported beam members to get the strain on each load stage, thereby obtaining sectional curvature distribution along the beam length.Specific specimen parameters, loading equipment, test phenomena etc. see reference [11].

According to the measured results of this paper, we gained the curvature distributions shown in figure 1 and figure 2.Seen from the figure that in terms of pure bending plastic hinge, with the sample size increases, the corresponding state for plastic hinge to form significantlyis more close to the late period of loading. 

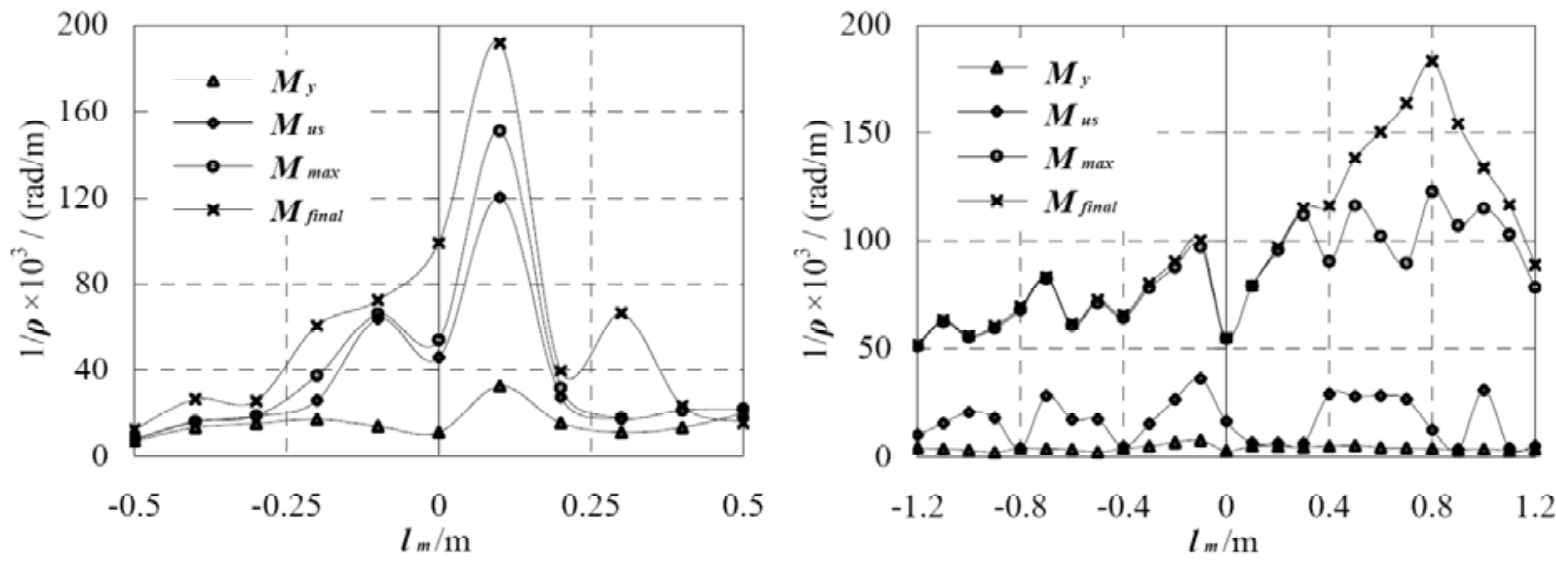

Fig.1 JD2-3 curvature distributionFig.2 JD5-1 curvature distribution

\section{Pure Bending Plastic Hinge Size Effect Analysis}

Parameter Analysis Considering the Size Effect of Pure Bending Plastic Hinge Contrasting the formation process of pure bending plastic hinge on different size components, the plastic hinge forms earlier for small size components.Non-elastic curvature significantly concentrate in a plastic hinge region in the limit and maximum load states,the equivalent length of plastic hinge region $l_{p}$ corresponds to beam height $h$, and the inelasticrotation angles under two loading states differs little,theseare basically consistent with the existing plastic hinge theory.

To maintain the calculation method on consistency, we can still assume that the equivalent length $l_{p}$ is equal to cross section height $h$.Then,in the ultimate bearing state, sectional stiffness on plastic hinge zone is multiplied by reduction coefficient $b_{p-u s}$, equivalent plastic corner is multiplied by increscent coefficient $t_{p-u s}$; in maximum load state, sectional stiffness on plastic hinge zone is multiplied by reduction coefficient $b_{p-\max }$, equivalent plastic corner is multiplied by increscentcoefficient $t_{p \text {-max }}$.Adjustment coefficients of different size components refer to the related fitting formulas in reference[11].

Parameter Calculation Considering the Size Effect of Pure Bending Plastic Hinge.The equivalent length $l_{p}$ can be equal to cross section height $h$, then:

$$
l_{p, y-u s}=l_{p, y-\max }=h
$$

the curvatures $1 / \rho_{y}$ at yield load state, $1 / \rho_{u s}$ at limit load state and $1 / \rho_{\max }$ atmaximum load state are calculated respectively according to the curvature calculation formulas considering size effect in reference [11], then the plastic curvature calculation formulas considering the size effect are as follows:

$$
\frac{1}{\rho_{p, y-u s}}=\frac{1}{\rho_{u s}}-\frac{1}{\rho_{y}}, \frac{1}{\rho_{p, y-\max }}=\frac{1}{\rho_{\max }}-\frac{1}{\rho_{y}}(2)
$$

According to formulas (1) (2), , plastic rotation angle $\theta_{p}$ is calculated by means ofthe integral of inelastic curvature along the equivalent length of plastic hinge area $l_{p}$ as follows:

$$
\theta_{p, y-u s}=\frac{1}{\rho_{p, y-u s}} \cdot l_{p, y-u s}, \theta_{p, y-\max }=\frac{1}{\rho_{p, y-\max }} \cdot l_{p, y-\max }
$$

Figure 3 is the calculated non-elastic curvature according to formula (2), Figure 4 is the calculated inelastic rotation angle according to formula (3). The results show that both inelastic curvature and non-elastic corner, with the sample size increases, the calculated value under maximum load condition significantly increases compared with that under bearing state.Further, in the ultimate 
bearing state, non-elastic corner dcreases slightly with increasing size; in maximum load state, non-elastic corner has a significant increase in the trend with specimen size increases. This is consistent with the observed trend.
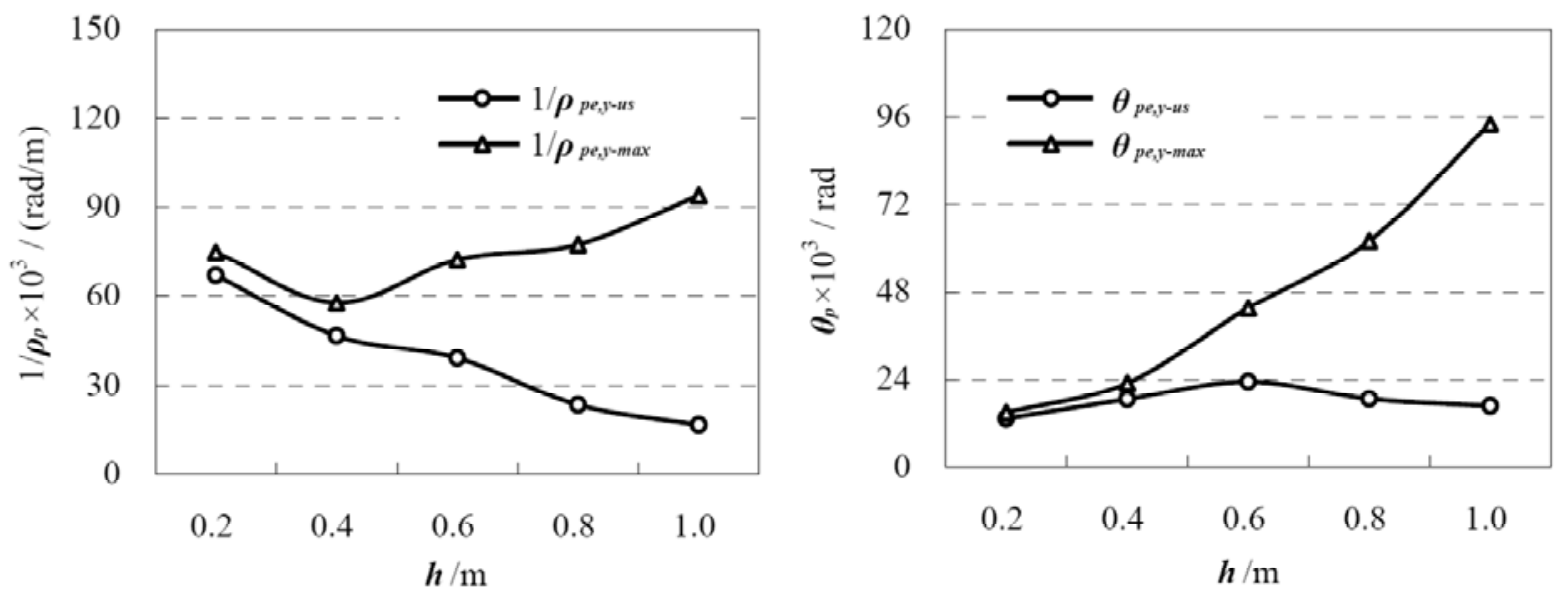

Fig.3 Plastic curvity calculated valueFig.4 Plastic rotation-angle measured value

Contrast Between Calculated Values and Measured Values of Pure Bending Plastic Hinge Parameters. Figure 5 and table 1 is a comparison between plastic curvature calculated values and measured values. Wherein, $1 / \rho_{p t, y-u s}$ and $1 / \rho_{p t, y-\text { mas }}$ are the plastic curvature measured values under the limit state and maximum load state; $1 / \rho_{p e, y-u s}$ and $1 / \rho_{p e, y-m a s}$ are the calculated values under the limit state and maximum load state; $\Delta_{y-u s, e-t}$ means the difference value between calculated and measured values. Figure 6and table 2 is a comparison of plastic corner calculated values and measured values, the subscript means the same as above. Comparative data show that using the calculation method proposed in this paper, in addition to the JD1 specimen under ultimate bearing state and JD3 under maximum load condition, the error is less than $10 \%$. Thecalculated curve and measured curve are basicallyconsistent.
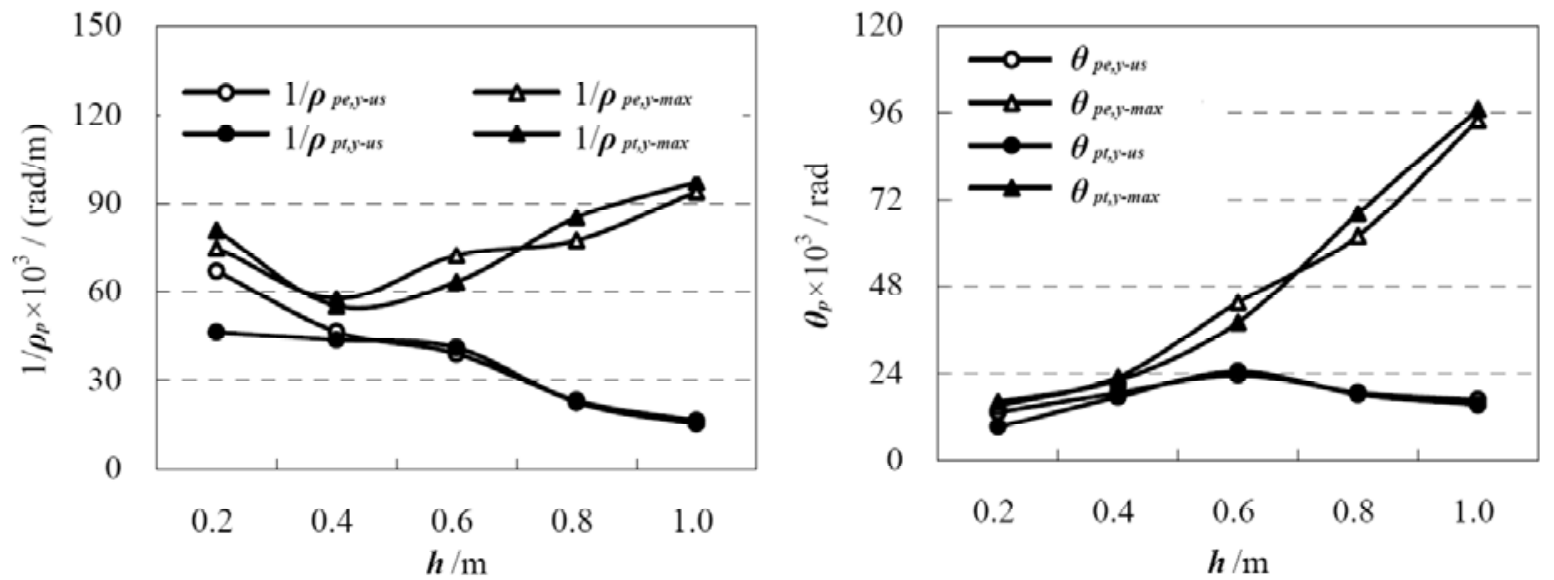

Fig.5 Plastic curvityFig.6 Plastic rotation-angle

Table 1 Plastic curvity measured and calculated value

\begin{tabular}{ccccccc}
\hline h/m & $\begin{array}{c}1 / \rho_{p t, y-u s} \\
/(\mathrm{rad} / \mathrm{m})\end{array}$ & $\begin{array}{c}1 / \rho_{p e, y-u s} \\
/(\mathrm{rad} / \mathrm{m})\end{array}$ & $\Delta_{y-u s, e-t}$ & $\begin{array}{c}1 / \rho_{p t, y-\max } \\
/(\mathrm{rad} / \mathrm{m})\end{array}$ & $\begin{array}{c}1 / \rho_{p e, y-\max } \\
/(\mathrm{rad} / \mathrm{m})\end{array}$ & $\Delta_{y-\max , e-t}$ \\
\hline 0.2 & 0.0464 & 0.06702 & $44.44 \%$ & 0.08083 & 0.07488 & $-7.37 \%$ \\
0.4 & 0.04373 & 0.04656 & $6.46 \%$ & 0.05517 & 0.05762 & $4.43 \%$ \\
0.6 & 0.04105 & 0.03907 & $-4.82 \%$ & 0.06341 & 0.07244 & $14.25 \%$ \\
0.8 & 0.02277 & 0.02336 & $2.57 \%$ & 0.08513 & 0.07753 & $-8.93 \%$ \\
1 & 0.01538 & 0.01674 & $8.81 \%$ & 0.09707 & 0.09391 & $-3.25 \%$ \\
\hline
\end{tabular}


Table 2 Plastic rotation-angle measured and calculated value

\begin{tabular}{ccccccc}
\hline$h / \mathrm{m}$ & $\theta_{p t, y-u s} / \mathrm{rad}$ & $\theta_{p e, y-u s} / \mathrm{rad}$ & $\Delta_{y-u s, e-t}$ & $\theta_{p t, y-\max } / \mathrm{rad}$ & $\theta_{p e, y-\max } / \mathrm{rad}$ & $\Delta_{y-\max , e-t}$ \\
\hline 0.2 & 0.00928 & 0.0134 & $44.44 \%$ & 0.01617 & 0.01498 & $-7.37 \%$ \\
0.4 & 0.01749 & 0.01862 & $6.46 \%$ & 0.02207 & 0.02305 & $4.43 \%$ \\
0.6 & 0.02463 & 0.02344 & $-4.82 \%$ & 0.03804 & 0.04346 & $14.25 \%$ \\
0.8 & 0.01822 & 0.01869 & $2.57 \%$ & 0.06811 & 0.06202 & $-8.93 \%$ \\
1 & 0.01538 & 0.01674 & $8.81 \%$ & 0.09707 & 0.09391 & $-3.25 \%$ \\
\hline
\end{tabular}

\section{Conclusions}

(1) The pure bending plastic hinge exhibited features on large size members are different from that on small size members, that is, when plastic hinges is significantly formed on lagrge size member, the corresponding loading status may have gone beyond the limit or the maximum load state.

(2)In order to maintain consistency on the calculation method ,for large-size pure bending member, inelastic deformation is artificially concentrated in the assumed plastic hinge region in plastic deformation calculation process , and thestiffness and equivalent corner adjustment coefficients for different size members are gotby date fitting, measured data and calculated results are in good agreement.

\section{Acknowledgements}

This work was financially supported by National Natural Science Foundation of China (51378030), Beijing Natural Science Foundation of China(8152004), Beijing Municipal Education Commission Research Project (km201410005019) and National Science \& Technology Pillar Program of China (2013BAJ10B03-02).

\section{References}

[1] XuemeiZhao ect. EngineeringStructures, 2012, 34:466 482.

[2] Zhigang Song ect. Journal ofBuilding Structures, 2009, 30(2):182 190.

[3] Yushen Qiu. Architecture Knowledge, 2007, 10:16 17(in Chinese)

[4] Zhongfeng Wei. Research \& Application of Building Materials, 2002, 4:39 40(in Chinese)

[5] Lian Duan, Wenchang Wang, Sukai Guo. Building Science Research of Sichuan, 1983, 3:16 22(in Chinese)

[6] Xingwu Zhai, Lunchang Yu,Li Zheng . Design of Water Resources \&Hydroelectric Engineering, 2002, 21(1):21 23(in Chinese)

[7] Yue Jiang, Xiaozu Su. Industrial Construction, 2008, 38:425 430(in Chinese)

[8] Y.J. Jin and B. Li. Journal of Wuhan University of Technology, 2010, 32(11):58-61(in Chinese)

[9] J.J. Wu. Journal of Railway Engineering Society, 2009, 5: 44-49(in Chinese)

[10]Ministry of Construction P R China. GB50010-2002 Code for Design of Concrete Structures[S]. Beijing: ChinaBuilding Industry Press, 2002(in Chinese)

[11]Hongyu Zhou. Test Study on Size Effect of Flexural Behavior of RC Flexural Members[D].Beijing:College of Architecture and Civil Engineering, Beijing University of Technology,2012 (in Chinese) 